\title{
OBSERVATION OF BERNSTEIN WAVES EXCITED BY NEWBORN INTERSTELLAR PICKUP IONS IN THE SOLAR WIND
}

\author{
Colin J. Joyce ${ }^{1}$, Charles W. Smith ${ }^{1}$, Philip A. Isenberg ${ }^{1}$, S. Peter Gary ${ }^{2}$, Neil Murphy ${ }^{3}$, \\ Perry C. Gray ${ }^{4}$, and LeOnard F. Burlaga ${ }^{5}$ \\ ${ }^{1}$ Physics Department, Space Science Center, University of New Hampshire, Durham, NH, USA; \\ cj146@unh.edu, Charles.Smith@unh.edu, Phil.Isenberg@unh.edu \\ ${ }^{2}$ Los Alamos National Laboratory, Los Alamos, NM, USA; pgary@lanl.gov \\ ${ }^{3}$ Jet Propulsion Laboratory, Mail Stop 180-600, 4800 Oak Grove Drive, Pasadena, CA, USA; Neil.Murphy@jpl.nasa.gov \\ ${ }^{4}$ P.O. Box 790, Los Alamos, NM, USA; Perry.Gray@dtra.mil \\ ${ }^{5}$ NASA/Goddard Space Flight Center, Geospace Physics Laboratory, Code 673, Greenbelt, MD 20771, USA; lburlagahsp@ verizon.net \\ Received 2011 October 18; accepted 2011 December 6; published 2012 January 6
}

\begin{abstract}
A recent examination of $1.9 \mathrm{~s}$ magnetic field data recorded by the Voyager 2 spacecraft in transit to Jupiter revealed several instances of strongly aliased spectra suggestive of unresolved high-frequency magnetic fluctuations at 4.4 AU. A closer examination of these intervals using the highest resolution data available revealed one clear instance of wave activity at spacecraft frame frequencies from 0.2 to $1 \mathrm{~Hz}$. Using various analysis techniques, we have characterized these fluctuations as Bernstein mode waves excited by newborn interstellar pickup ions. We can find no other interpretation or source consistent with the observations, but this interpretation is not without questions. In this paper, we report a detailed analysis of the waves, including their frequency and polarization, that supports our interpretation.
\end{abstract}

Key words: cosmic rays - magnetic fields - turbulence - waves

\section{INTRODUCTION}

In the following, we describe observations by the Voyager 2 magnetometer (Behannon et al. 1977) at $4.4 \mathrm{AU}$ of waves with linear polarization and spacecraft frame frequencies significantly greater than the proton cyclotron frequency when the mean interplanetary magnetic field (IMF) $\mathbf{B}_{0}$ is perpendicular to the solar wind flow velocity. There are several potential sources and interpretations for these waves, and each source implies specific wave characteristics that can be tested, but most sources and interpretations can be reliably excluded. At these frequencies, the two most likely plasma modes are whistler and Bernstein modes. Direct and unambiguous identification of the wave properties is difficult, but with the observed linear polarization and a strongly oblique IMF we argue that propagation at large angles to the IMF is likely. These facts, together with the observed spacecraft frame frequencies, strongly point to the Bernstein mode as the probable identity of the waves. With no other recognized discrete source for energetic particles, we conclude that the likeliest source is newborn interstellar pickup particles, probably $\mathrm{H}^{+}$. We argue that these two conclusions (source and mode identification) are consistent and expected given the local plasma conditions. If we are correct, it is possible that Bernstein mode waves are a common and previously unappreciated feature of the outer heliosphere.

Interplanetary magnetic waves arising from wave/particle interactions most normally fall into several classes. Lowfrequency Alfvén or fast-mode waves frequently arise due to field-aligned and gyrating beams of energetic ions and are observed to be strongly polarized, parallel propagating in the same direction as the ion beam, and with magnetic fluctuations perpendicular to the mean IMF (Gary et al. 1984; Murphy et al. 1995; Lee \& Ip 1987; Isenberg 2005; Joyce et al. 2010). Higher frequency waves can arise from field-aligned electron beams or gyrating ion distributions. For electron beams the dominant instability is the parallel-propagating and highly polarized whistler mode (Wong \& Smith 1994). However, gyrating ion beams can produce whistler waves at small to modest angles of propagation relative to the mean IMF (Wong \& Goldstein 1987, 1988). Velocity ring ion distributions due to ion pickup at solar wind velocities perpendicular to $\mathbf{B}_{0}$ are more likely to produce linearly polarized Bernstein waves propagating at large angles to the mean IMF (Perraut et al. 1982; McClements \& Dendy 1993; McClements et al. 1994; Gary et al. 2010, 2011; Liu et al. 2011).

Bernstein waves are not commonly discussed in the solar wind literature. For this reason, our early working premise was that the observed waves were the result of a high-frequency instability described by Gray et al. (1996) whereby newly ionized interstellar pickup ions (PUIs) excite whistler waves with large growth rates. However, the whistler waves described by Gray et al. are circularly polarized whereas the observations we will describe are linearly polarized. Circular polarization is consistent with wave propagation in a single direction and at relatively small angle with respect to the mean magnetic field $\mathbf{B}_{0}$. We observe these waves at times when $\mathbf{B}_{0}$ is perpendicular to the flow, which means that there is no preferred direction for a PUI source and wave propagation in both directions would be expected. At the very least, the observed linear polarization suggests either a significant balance between whistler waves propagating in both directions or a large angle between the wave vectors $\mathbf{k}$ and $\mathbf{B}_{0}$. Such a high degree of balance seems unlikely without a local scattering mechanism. Whistler waves propagating at large angles to $\mathbf{B}_{0}$ can be elliptical to nearly linearly polarized, but they show magnetic fluctuations with strong components along $\mathbf{B}_{0}$. Our observations show little to no projection of the magnetic fluctuation along $\mathbf{B}_{0}$ in the early stage of the observations, and then develop significant projection as the event progresses.

In the final analysis, using wave frequency and polarization, we conclude that the observed magnetic fluctuations are not whistler waves. Instead, we believe these waves are due to an 
ion Bernstein mode instability driven by an ion ring velocity distribution which is characteristic of interstellar PUIs at solar wind velocity perpendicular to $\mathbf{B}_{0}$. The enhanced magnetic fluctuations resulting from such an instability are linearly polarized and propagate at large angles to $\mathbf{B}_{0}$ (Gary et al. 2011; Liu et al. 2011). They also show strong projections of $\delta \mathbf{B}$ onto $\mathbf{B}_{0}$ when the plasma $\beta$ is high. If we are correct in attributing the source of these waves to be newborn interstellar PUIs interacting with a perpendicular magnetic field, then these waves should be a common feature of the outer heliosphere wherever hydrogen atom ionization is prevalent.

\section{DATA ANALYSIS}

We have studied two years of Voyager 2 magnetic field data (Behannon et al. 1977) spanning the range from 1 to $4.4 \mathrm{AU}$ in search of low-frequency waves excited by interstellar PUIs. Although theoretical treatments would suggest that such lowfrequency PUI-excited waves are common (Lee \& Ip 1987), we found only one such event where waves were seen resulting from both pickup $\mathrm{H}^{+}$and $\mathrm{He}^{+}$ions and published that analysis in a companion paper (Joyce et al. 2010). Unlike the observations reported here, the IMF was highly radial during this lowfrequency event. The relative scarcity of such events may be due to the spacecraft location within the hydrogen cavity and the lower density of He atoms. We generated power spectra using an analysis technique described in the companion paper and searched the spectra for wave enhancements at spacecraft frame frequencies comparable to the proton cyclotron frequency and indicative of PUI activity. It is important to note that the data used in that study had insufficient time resolution to resolve the waves reported here. However, during this process we occasionally observed spectra that turn up strongly at high frequencies. This turn up in many cases is stronger than what is normally expected from data aliasing and may be indicative of spectral features at unresolved higher frequencies. Using these events as a guide, we obtained three days of the highest time resolution magnetic field data available $(0.3 \mathrm{~s})$ and repeated our analyses with various subsets of the days in question. It should be noted that the $0.3 \mathrm{~s}$ MAG data from Voyager has not been extensively surveyed for interplanetary physics while in cruise mode. In those three days we found one protracted interval of high-frequency wave activity that extended from 20:00 to 22:00 UT on day 350 of 1978. The waves were seen at spacecraft frequencies $0.1<v_{\mathrm{sc}}<1.5 \mathrm{~Hz}$ and are analyzed below. Without an exhaustive survey, the actual frequency of occurrence of these waves is unknown. We speculate that the waves may be a common feature of the outer heliosphere only because the source is thought to be present and steady.

During the event considered in this manuscript, Voyager 2 was at 4.4 AU and 199 days before the inbound crossing of the Jovian bow shock. Jupiter is at -5.43 relative longitude from the spacecraft and 1.29 relative latitude at a relative distance $0.8 \mathrm{AU}$. Because of this, we must also consider the possibility that any wave activity seen at this time may result from Jovian ions or electrons. Figure 1 shows the magnetic field conditions and solar wind parameters for day 350 of 1978. The magnetic field is shown in $(\mathbf{R}, \mathbf{T}, \mathbf{N})$ coordinates where $\mathbf{R}$ is the unit vector pointing radially outward from the Sun to the observation point, $\mathbf{T}$ is the unit vector coplanar with the rotational equator of the Sun and directed in the sense of positive rotation, and $\mathbf{N}$ is defined by $\mathbf{N}=\mathbf{R} \times \mathbf{T}$. We define the IMF latitude as the angle $\delta=\arctan \left(\mathbf{B}_{N} /\left(\mathbf{B}_{R}^{2}+\mathbf{B}_{T}^{2}\right)^{1 / 2}\right)$ between the IMF vector and the $(\mathbf{R}, \mathbf{T})$ plane with positive latitude in the sense of increasing $B_{N}$.

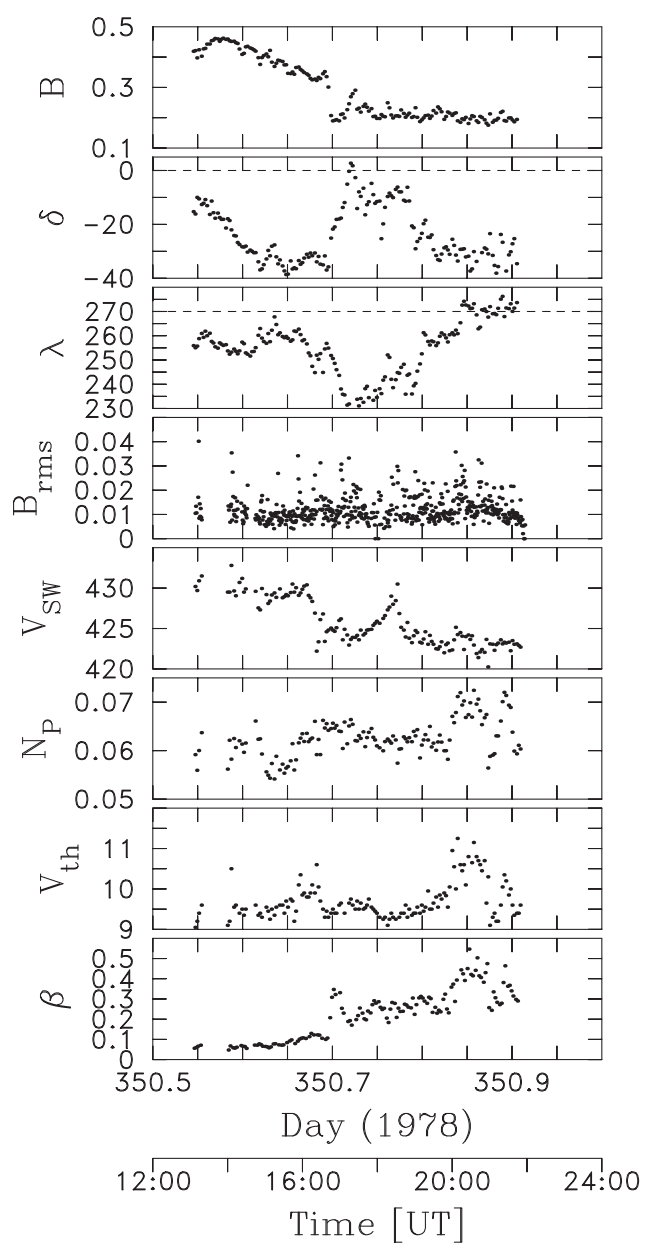

Figure 1. Second half of day 350 showing (top to bottom) IMF intensity B (nT), IMF latitude angle $\delta$ (deg), IMF longitude angle $\lambda$ (deg), rms fluctuation level for $0.3 \mathrm{~s}$ measurements relative to a $48 \mathrm{~s}$ mean $B_{\mathrm{rms}}(\mathrm{nT})$, solar wind speed $V_{\mathrm{SW}}\left(\mathrm{km} \mathrm{s}^{-1}\right)$, proton density $N_{p}\left(\mathrm{~cm}^{-3}\right)$, proton thermal speed $v_{\text {th }}\left(\mathrm{km} \mathrm{s}^{-1}\right)$, and $\beta_{p}$. Values are averages over four consecutive data points at $48 \mathrm{~s}$ resolution each producing averages at $192 \mathrm{~s}$ resolution. The dashed lines in panels 2 and 3 denote the perpendicular IMF geometry, nearest to the expected Parker spiral orientation, that favors the Bernstein instability. Both decimal day and UT are shown at the bottom.

The longitude is the angle $\lambda=\arctan \left(\mathbf{B}_{T} / \mathbf{B}_{R}\right)$ in the $(\mathbf{R}, \mathbf{T})$ plane between the IMF vector and the $\mathbf{R}$ direction with the angle increasing in the sense of increasing $\mathbf{B}_{T}$. At this distance the nominal Parker spiral direction would be $\lambda=280^{\circ}\left(-80^{\circ}\right)$ for an away sector and $100^{\circ}$ for a toward sector with $\delta=0^{\circ}$ for both. The magnitude of the IMF is descending from $\sim 0.5 \mathrm{nT}$ to $\sim 0.2 \mathrm{nT}$ from 350.55 to $350.75 \mathrm{UT}$. The IMF is nearly azimuthal and southward pointing until $350.65 \mathrm{UT}$, and then turns $40^{\circ}$ from the azimuthal direction $\left(50^{\circ}\right.$ from the nominal spiral direction) while at the same time losing its southward component, until $\sim 350.8$ UT when it resumes a more normal spiral orientation perpendicular to the radial direction and at the same time regains its southward component. Magnetic connection to Jupiter is improbable except possibly from 350.7 to 350.8 UT. It never gains a radial orientation, but it does reach $\sim 50^{\circ}$ from radial at $\sim 350.72$ UT. For the remainder of the time plotted the IMF is more nearly $90^{\circ}$ from the Jovian direction. Magnetic connection to Jupiter or its bow shock is never likely and we will discuss in Section 3 additional reasons why the observed waves are probably not whistlers originating from Jovian electrons.

The solar wind speed, density, and temperature demonstrate only small changes during the half-day plotted, but we note 


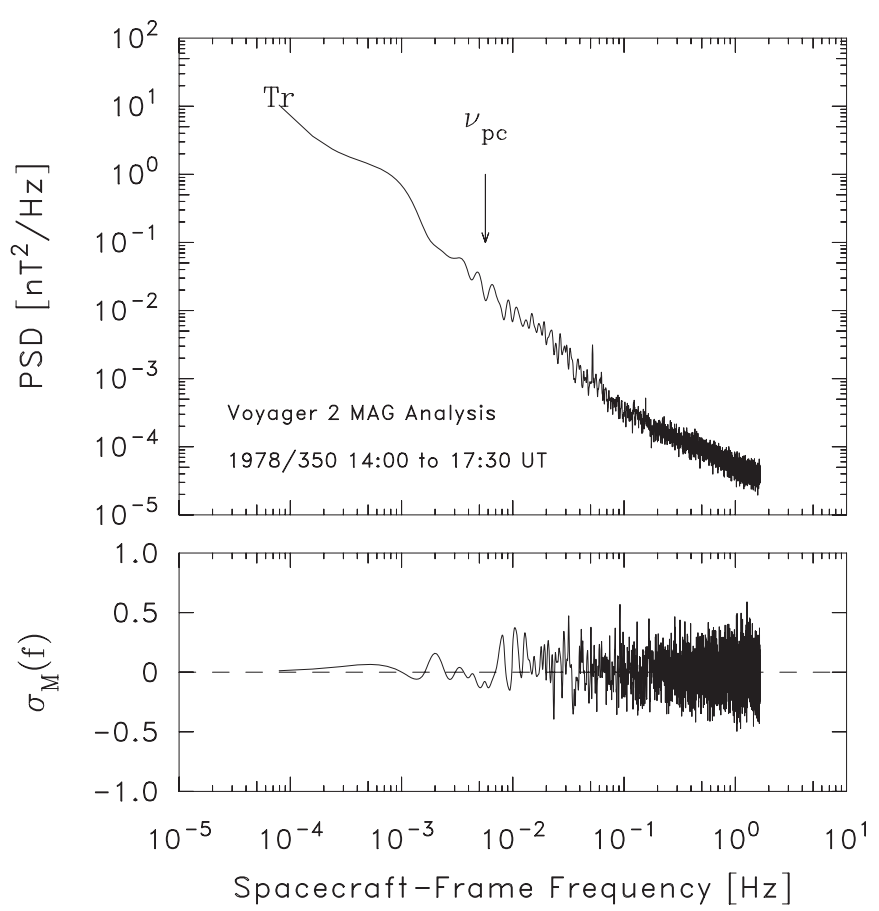

Figure 2. Top: computed power spectrum trace (marked "Tr") for IMF fluctuations using 0.3 s data for day 350 14:00 to 17:30 UT. The proton cyclotron frequency $v_{\mathrm{pc}}$ is marked for reference. Bottom: computed normalized magnetic helicity spectrum for the same period. This is a good example of an undisturbed IMF spectrum before and after a wave event.

that the interval when the waves in question are observed runs from 350.83 to 350.92 UT. During this time the IMF intensity is constant, the IMF direction is relatively constant, the wind speed $V_{\mathrm{SW}}$ is constant at $422 \mathrm{~km} \mathrm{~s}^{-1}$, but the proton density $N_{P}$ and temperature $T_{P}$ are elevated. This elevation of density and temperature seems highly correlated with the observation of the waves. Throughout the half-day plotted $\beta_{p}=8 \pi N_{P} k_{B} T_{P} /|B|^{2}$ is rising and peaks over the same period as the observed wave activity.

The observation comes eight days into a 13 day rarefaction interval spanning days 342 through 355 where the wind speed decreases from a maximum of $\sim 700 \mathrm{~km} \mathrm{~s}^{-1}$ to a minimum of $\sim 350 \mathrm{~km} \mathrm{~s}^{-1}$ with $V_{\mathrm{SW}} \sim 420 \mathrm{~km} \mathrm{~s}^{-1}$ at the time of the wave activity. This is the traditional type of rarefaction that does not produce strongly radial magnetic fields. The proton density decreases more rapidly during the first two days (not shown) and thereafter is constant at $\sim 0.08-0.1 \mathrm{~cm}^{-3}$ for 12 days including the time of observation. The proton temperature decreases similarly and remains constant at $\sim 10^{4} \mathrm{~K}$ from the start of day 350 through day 355 while $\beta_{p}$ varies from $\sim 0.3$ to $\sim 0.5$ during the time of the waves having risen from a minimum of $\sim 0.05$ at the start of Figure 1 . The behavior of $\beta_{p}$ together with the orientation of the IMF plays a key role in when the waves are observed and we will return to this point below.

The IMF direction during the eight days prior to the wave event shows good agreement with the Parker prediction for an away sector at this distance with occasional north/south deflections. Following the wave event and starting midday on DOY 351 there is three-day period of apparent current sheet crossings when the polarity of the hourly averages is variable. The entire rarefaction interval ends with a possible shock crossing on day 355. We do not believe these are waves originating with a shock-accelerated population, either, as magnetic connection to the shock seems unlikely.
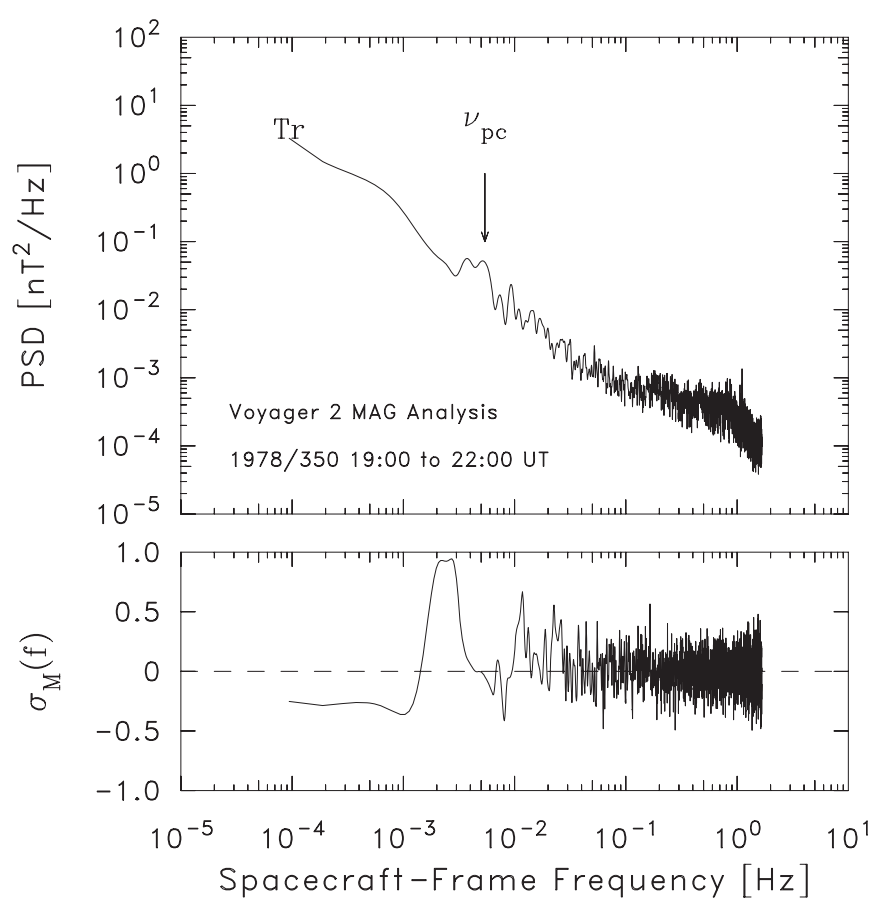

Figure 3. Top: computed power spectrum trace for IMF fluctuations using $0.3 \mathrm{~s}$ data for day 350 19:00 to 22:00 UT. Bottom: computed normalized magnetic helicity spectrum for the same period. Note the enhanced power level at high frequencies between 0.1 and $1.5 \mathrm{~Hz}$.

We contend that the waves presented here result from instabilities related to newborn interstellar pickup $\mathrm{H}^{+}$. At $r=4.4 \mathrm{AU}$ the Voyager spacecraft is inside the ionization cavity for interstellar hydrogen, the radial scale of which we take to be $L=5.6 \mathrm{AU}$. Assuming the density of hydrogen in interstellar space to be $N_{\mathrm{H}_{0}}=0.1 \mathrm{~cm}^{-3}$ (Gloeckler et al. 1997; Bzowski et al. 2009), we can compute the local density of neutral $\mathrm{H}$ to be $N_{\mathrm{H}}=N_{\mathrm{H}_{0}} e^{-L / r}=0.028 \mathrm{~cm}^{-3}$. Interstellar neutral $\mathrm{H}$ is ionized via charge exchange with solar wind ions as well as photoionzation. The charge exchange ionization rate is given by $\beta_{\mathrm{ce}}=\sigma_{\mathrm{H}} N_{P} V_{\mathrm{SW}}$ where $\sigma_{\mathrm{H}}$ is the cross section of hydrogen $\left(2 \times 10^{-15} \mathrm{~cm}^{2}\right)$. Using $N_{P}=0.07 \mathrm{~cm}^{-3}$ and $V_{\mathrm{SW}}=423 \mathrm{~km} \mathrm{~s}^{-1}$, we compute $\beta_{\mathrm{ce}}=5.92 \times 10^{-9} \mathrm{~s}^{-1}$. The photoionization rate is given by $\beta_{\mathrm{ph}}=\beta_{\mathrm{ph}}^{0} r^{-2}=5.17 \times 10^{-9} \mathrm{~s}^{-1}$ where $\beta_{\mathrm{ph}}^{0}=10^{-7} \mathrm{~s}^{-1}$ is the measured rate at $1 \mathrm{AU}$ (Ruciński et al. 1996). The resulting rate of newborn pickup $\mathrm{H}^{+}$production is given by $N_{\mathrm{H}}\left(\beta_{\mathrm{ce}}+\beta_{\mathrm{ph}}\right)=3.1 \times 10^{-10} \mathrm{p}^{+} \mathrm{cm}^{-3} \mathrm{~s}^{-1}$. We offer these numbers for perspective, but we will not be computing growth rates here.

Figure 2 shows the power and helicity spectra computed for 14:00 to $17: 30 \mathrm{UT}$ on day 350 . It is entirely indicative of periods before and after the wave period in question. The power spectrum (top) is smooth and featureless with a long tail extending to the Nyquist frequency. Figure 7 of Behannon et al. (1977) suggests that the measurement has reached the noise level of the instrument by $\sim 80 \mathrm{mHz}$ and the flattened region of the measured spectrum above this frequency is not a clean measurement of the IMF. The helicity spectrum (bottom) shows no uniformly biased frequency interval, but this is expected for the noise spectrum above $\sim 80 \mathrm{mHz}$ and says nothing significant of the IMF fluctuations at these frequencies.

Figure 3 (top) shows the spectral analysis of the interval 19:00 to 22:00 UT. We observe two broad, high-frequency spectral enhancements, at approximately 0.2 and $0.8 \mathrm{~Hz}$ that are rising 

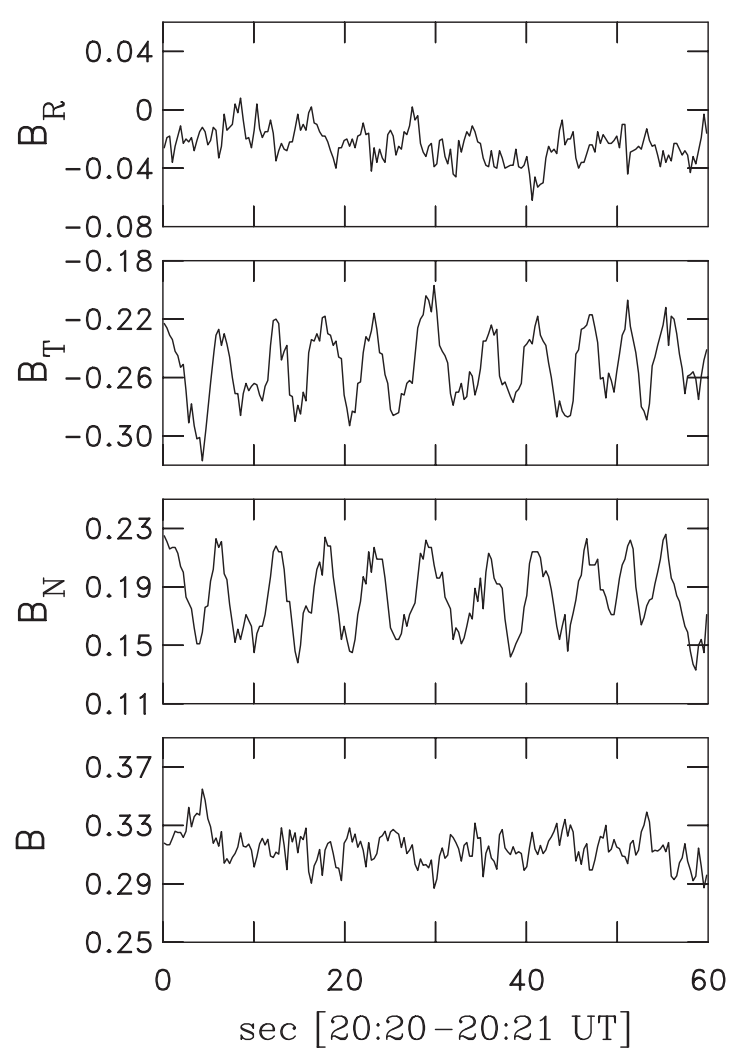

Figure 4. Plot of IMF during day 350 for one-minute interval 20:20 to 20:21 UT using highest resolution $0.3 \mathrm{~s}$ MAG data. With $\sim(5(1 / 2))$ oscillations in the latter $30 \mathrm{~s}$, the wave period in the spacecraft frame is $\sim 5.5 \mathrm{~s}$, so the spacecraft frame frequency is $\sim 0.18 \mathrm{~Hz}$. To the extent that the observed power spectrum appears to exhibit two broad peaks of enhanced wave activity, the waves in this time interval are consistent with the lower frequency peak at $\sim 0.2 \mathrm{~Hz}$.

above the measured noise level. We will demonstrate below that Figure 3 does indeed represent several distinct wave forms with changing spacecraft frequencies and argue that changing plasma conditions can probably account for the observed frequency shifts. We also note a slight enhancement at the proton cyclotron frequency which could be due to pickup $\mathrm{H}^{+}$ions, but we find no strong evidence of low-frequency waves originating with newborn interstellar PUIs.

Figure 3 (bottom) shows the spectrum of normalized magnetic helicity which is the spectral form of the correlation:

$$
\sigma_{M} \equiv|k|\langle\mathbf{A} \cdot \mathbf{B}\rangle / E_{B}
$$

where $\mathbf{B}$ is the magnetic field, $\mathbf{A}$ is the vector potential, $E_{B}$ is the magnetic energy, and $\left|\sigma_{M}\right| \leqslant 1$. Note that $\sigma_{M} \sim 0$ over the frequency range $0.2-1 \mathrm{~Hz}$. This event shows no helicity enhancement and as a result it shows no polarization in the spacecraft frame (Smith et al. 1984). Because the power rises above the background noise level, it is expected that a polarization signature would be evident if it were present, even if it were somewhat diluted by the background. Electron beams originating with either Jupiter or the downstream shock would produce whistler waves with a definite circular polarization as the resonance condition would require that the waves be Sunward propagating. Bernstein waves are linearly polarized and consistent with the $\sigma_{M} \simeq 0$ measurement.

Figure 4 shows the time series for a one minute subset of the wave period. The plot runs from 20:20 to 20:21 UT (350.8472 to 350.8479 ) and the mean field is clearly in the (T, N) plane. A nearly monochromatic wave can be observed in the $\mathbf{T}$ and

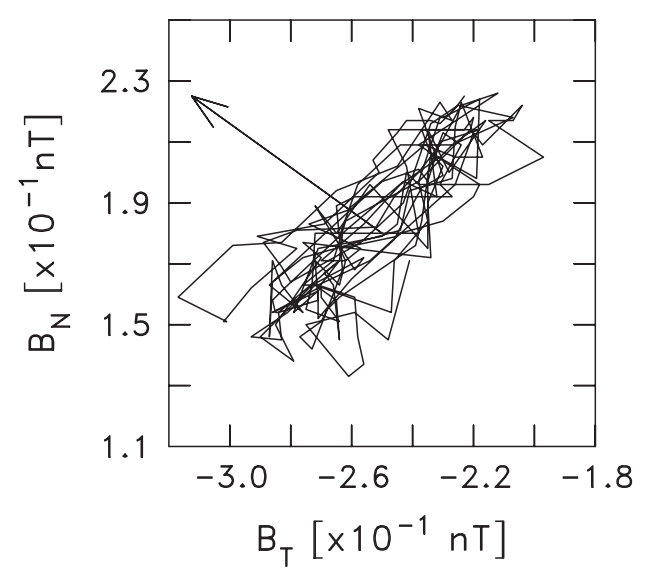

Figure 5. Hodogram of the $T$ and $N$ components of the IMF fluctuation for the same time interval and data resolution shown in Figure 4. The arrow indicates the direction of the mean magnetic field.

$\mathbf{N}$ components at a spacecraft frame period $\sim 5 \mathrm{~s}$ with little fluctuation in the field magnitude.

Figure 5 shows the hodogram of the same time interval in the $(\mathbf{T}, \mathbf{N})$ plane with the mean magnetic field direction marked by the arrow. Clearly, the fluctuations are perpendicular to the mean field direction and linearly polarized. Although Bernstein waves generally display a fluctuation component along the mean field, they do not at low $\beta$. In contrast, the whistler wave is an extension of the fast mode wave and develops linear polarization for high angles of propagation relative to the mean magnetic field and at the same time displays significant projection of the fluctuation onto the mean field. Their fluctuation direction remains perpendicular to the wave vector $\mathbf{k}$, but have significant projections onto the mean field according to the angle between $\mathbf{B}_{0}$ and $\mathbf{k}$. In other words, linearly polarized whistler fluctuations are not expected to be transverse to the mean magnetic field as these wave fluctuations are seen to be.

Figure 6 shows a second one minute subset of the wave interval from 21:39 to 21:40 UT (350.9021 to 350.9028). The mean magnetic field is again in the $(\mathbf{T}, \mathbf{N})$ plane. The wave begins with spacecraft frame periods comparable to the previous example, but the wave period continually shortens until the wave shows half its original period by the end of the interval. This is a spacecraft-frame measurement, so we must caution that the observed change in wave period could result from changing Doppler shift conditions and may not be the result of changing plasma frame wave parameters. In this instance we observe a greater degree of fluctuation in the field intensity.

Figure 7 shows the $(\mathbf{T}, \mathbf{N})$ hodogram for this second oneminute interval. However, while the wave remains linearly polarized the observed fluctuation direction is no longer perpendicular to the mean field direction, but appears to have a significant projection onto the mean field. The projection of the fluctuation along the mean field accounts for the enhanced fluctuations in the magnitude of the field shown in Figure 6.

The waves observed during this two-hour period are seen to manifest both a changing spacecraft frame frequency and a changing angle of projection of the magnetic fluctuation onto the mean IMF. The one constant during the analysis is that significant magnetic fluctuations along the radial direction are never seen. Figure 8 (top) shows the measured wave frequency as a function of time throughout the wave interval. Vertical bars denote the range of frequencies observed within any given one-minute period. Note the changing wave period in the spacecraft frame. Figure 8 (bottom) shows $\Theta_{\mathbf{d B}, \mathbf{B}_{0}}$, the computed 

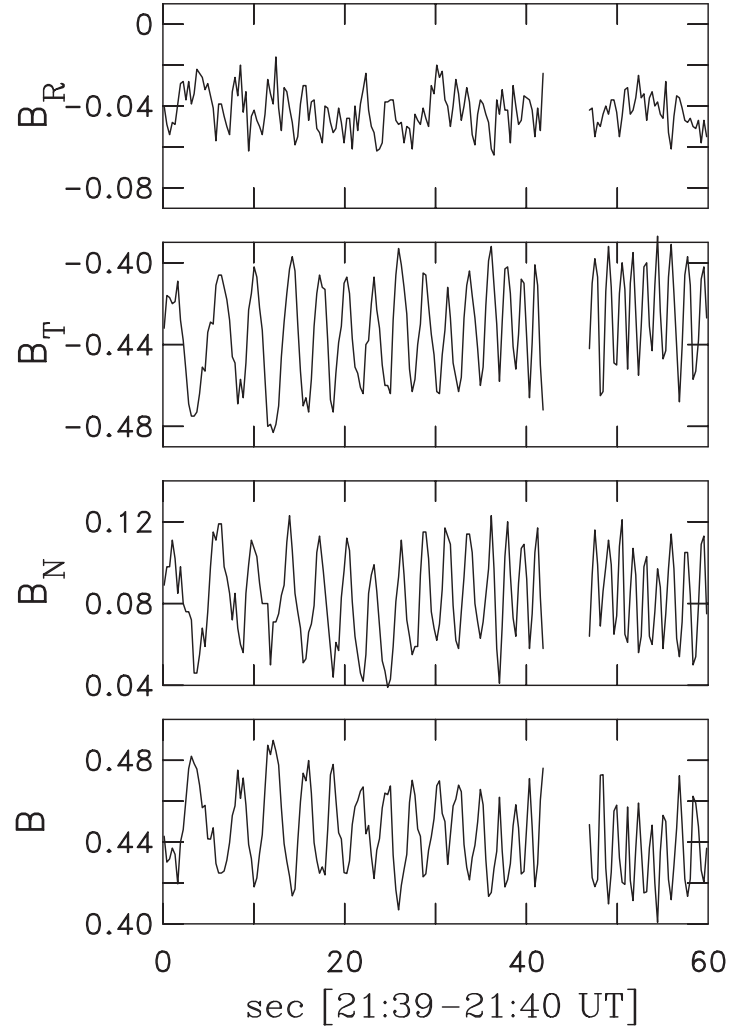

Figure 6. Plot of IMF during day 350 for one-minute interval 21:39 to 21:40 UT. Again, $0.3 \mathrm{~s}$ MAG data are plotted. While the minute begins with waves showing spacecraft frame periods $\sim 5 \mathrm{~s}$, the waves evolve through $\sim 3 \mathrm{~s}$ periods until in the last $15 \mathrm{~s}$ of the minute they exhibit periods $\sim 1.5 \mathrm{~s}$. This means a spacecraft frame frequency of $0.67 \mathrm{~Hz}$ which is well within the second broad wave peak at $\sim 0.8 \mathrm{~Hz}$ in the above spectrum.

angle between the magnetic fluctuations and the mean field for that same one-minute period. While the waves are seen to be transverse to the mean IMF at the beginning of the twohour interval, they evolve beginning 350.87 UT to develop a significant projection onto the mean IMF.

\section{THEORY}

Consider a homogeneous, magnetized, collisionless solar wind plasma which flows with velocity $\mathbf{V}_{\text {Sw }}$ perpendicular to a uniform magnetic field $\mathbf{B}_{0}$. If there is a background stationary neutral gas and an ionization mechanism (e.g., ionizing radiation or weak-charged-neutral collisions) present, the newborn ions will be picked up by the plasma flow and form an initially cold velocity-ring distribution. The resulting overall distribution, consisting of the tenuous velocity-ring component and the more dense, thermal solar wind component, is unstable to two distinct categories of instabilities.

One category is that of low-frequency electromagnetic cyclotron instabilities where the wave frequency in the plasma frame, $\omega_{r}$, satisfies $\omega_{r} \lesssim \Omega_{p}$, maximum growth rate at $\mathbf{k} \times \mathbf{B}_{0}=0, \delta E_{\|}=\delta B_{\|} \stackrel{=}{=}$, and circularly polarized $\delta E_{\perp}$ and $\delta B_{\perp}$. If the velocity ring is cold, $\omega_{r} \simeq \Omega_{p}$ (Wu \& Davidson 1972 ), whereas if the ring is broadened in velocity, the instability has Alfvén-cyclotron dispersion (Gary \& Madland 1988). In any case, simulations show that the primary consequence of the wave-particle interactions from enhanced field fluctuations driven by such instabilities is pitch-angle scattering of the velocity-ring ion component (Gary et al. 1986, 1988; Florinski et al. 2010).

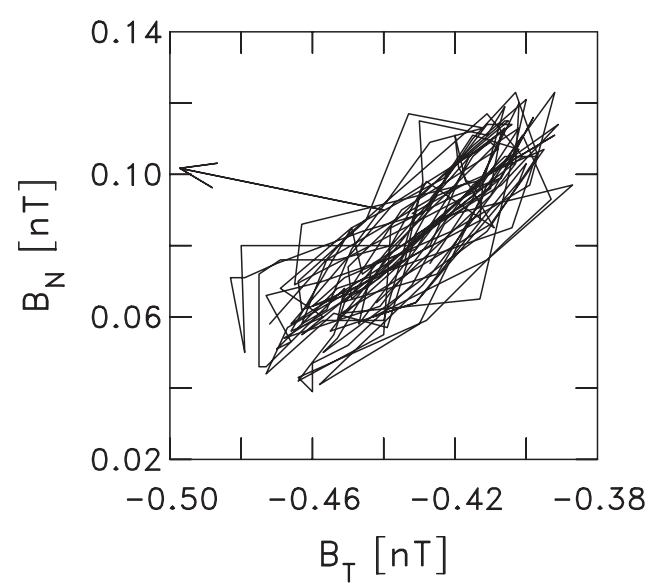

Figure 7. Hodogram of the $T$ and $N$ components of the IMF fluctuation for the same time interval and data resolution shown in Figure 6. The arrow indicates the direction of the mean magnetic field.
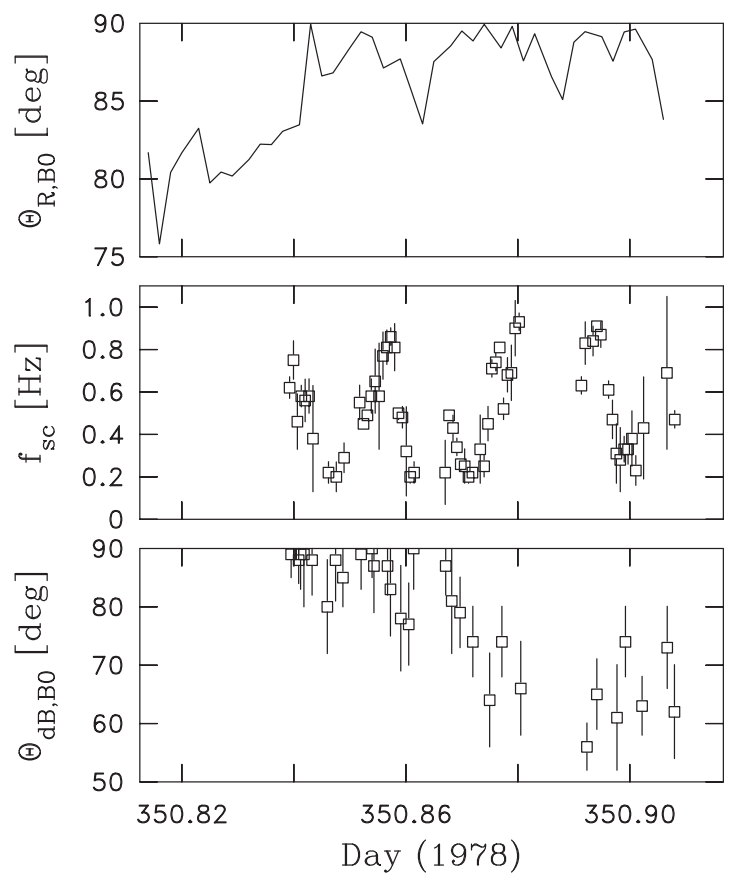

Figure 8. Top: angle between the radial direction and the mean IMF $0 \leqslant$ $\Theta_{\mathbf{R}, \mathbf{B}_{0}} \leqslant 90^{\circ}$. Middle: spacecraft frame frequency of observed wave forms for each $1 \mathrm{~m}$ interval. Vertical uncertainty denotes the range of frequencies observed within that minute of data. Bottom: angle between the magnetic fluctuation and the mean magnetic field $\Theta_{\mathbf{d B}, \mathbf{B}_{0}}$ defined to be between $0^{\circ}$ and $90^{\circ}$. Vertical lines denote estimated uncertainty in $\Theta_{\mathbf{d B}, \mathbf{B}_{0}}$.

The second category of instabilities driven by ion velocityring distributions is Bernstein mode instabilities. Linear theory predicts that such unstable modes generally follow magnetosonic/whistler dispersion, but are excited only near harmonics of the proton cyclotron frequency $\Omega_{p}$ with maximum growth rates at wavevector $\mathbf{k}$ almost perpendicular to $\mathbf{B}_{0}$ (Perraut et al. 1982; McClements \& Dendy 1993; McClements et al. 1994). These Bernstein mode instabilities typically have an appreciable compressive magnetic component $\left(\delta B_{\|} \neq 0\right)$ and are linearly polarized in the plane perpendicular to $\mathbf{B}_{0}$. Furthermore, their fluctuating electric fields are predominantly electrostatic, that is, $\delta \mathbf{E}$ is approximately parallel to the wavevector $\mathbf{k}$ (Gary et al. 2010, 2011). Another important property of proton Bernstein instabilities driven by ring velocity distributions is 
Table 1

Solar Wind Parameters

\begin{tabular}{lc}
\hline \hline Parameter & Value \\
\hline$|B|$ & $0.2 \mathrm{nT}$ \\
$V_{\mathrm{SW}}$ & $423 \mathrm{~km} \mathrm{~s}^{-1}$ \\
$N_{P}$ & $0.07 \mathrm{~cm}^{-3}$ \\
$v_{p, \mathrm{th}}$ & $10.5 \mathrm{~km} \mathrm{~s}^{-1}$ \\
$T_{P}$ & $0.57 \mathrm{eV}$ \\
$\beta_{p}$ & 7 \\
$\Omega_{\mathrm{cp}}$ & $0.02 \mathrm{~s}^{-1}$ \\
$\Omega_{\mathrm{ce}}$ & $35.2 \mathrm{~s}^{-1}$ \\
$\omega_{\mathrm{pi}}$ & $349 . \mathrm{s}^{-1}$ \\
$\Omega_{\mathrm{LH}}$ & $0.84 \mathrm{~s}^{-1}$ \\
\hline
\end{tabular}

that they are favored by relatively low proton beta values; e.g., in Gary et al. (2011), maximum growth rate is at $\beta_{p} \simeq 0.10$.

There are only a few simulations of instabilities driven by PUI velocity ring distributions. Gray et al. (1996) inserted ions at high perpendicular velocities in a hybrid simulation to drive both an Alfvén-cyclotron instability at $\omega_{r}<\Omega_{p}$ and a highfrequency, circularly polarized mode at $\omega_{r} \simeq 32 \Omega_{p}$ which they concluded were whistlers. They argued that their simulated highfrequency mode was related to the oblique whistler instability described by the linear theory of Wong \& Goldstein (1988). Liu et al. (2011) used an ion velocity-ring distribution to excite Bernstein mode growth in a particle-in-cell simulation; the primary consequence of wave-particle scattering by the enhanced fluctuations is to broaden the width of the initially thin perpendicular velocity distribution. These simulations also confirm that these modes have a strong compressive magnetic fluctuation component and that the fluctuations are more nearly linearly than circularly polarized in the plane perpendicular to the background magnetic field.

\section{APPLICATION OF THEORY}

We combine the measured solar wind conditions during the observed wave activity as taken from Figure 1 with a series of plasma parameters relevant to whistler and Bernstein mode identification and list them in Table 1. We define $\Omega_{\mathrm{LH}}=$ $\left[\left(\Omega_{\mathrm{ci}} \Omega_{\mathrm{ce}}\right)^{-1}+\omega_{\mathrm{pi}}^{-2}\right]^{-1 / 2}$ to be the lower hybrid frequency, $\Omega_{\mathrm{ce}}=e|B| / m_{e} c$ to be the electron cyclotron frequency with $e$ and $m_{e}$ the charge and mass of the electron and $c$ the speed of light, $\Omega_{\mathrm{cp}}=e|B| / m_{p} c$ the proton cyclotron frequency with $m_{p}$ the proton mass, $\omega_{\mathrm{pi}}=\left(4 \pi N_{P} e^{2} / m_{p}\right)^{1 / 2}$ is the proton plasma frequency, $v_{e, \text { th }}$ and $v_{p \text {,th }}$ the electron and proton thermal speeds, $k_{\|}$and $k_{\perp}$ the components of the wave vector $\mathbf{k}$ parallel and perpendicular to the mean magnetic field, and $\delta B_{\|}$and $\delta B_{\perp}$ the magnetic fluctuation amplitude parallel and perpendicular to the mean magnetic field. Using these definitions, one can distinguish
Bernstein from whistler modes according to the properties listed in Table 2. For instance, it is questionable whether the observations ever achieve the whistler mode equality $\left(\delta B_{\|}\right)^{2} \simeq$ $\left(\delta B_{\perp}\right)^{2}$, but it is clear that the Bernstein mode inequality $\left(\delta B_{\|}\right)^{2}<\left(\delta B_{\perp}\right)^{2}$ is maintained throughout the observations.

We compute the thermal proton gyroradius from the proton thermal speed $\rho_{p}=v_{p, \mathrm{th}} / \Omega_{\mathrm{cp}} \simeq 330 \mathrm{~km}$. If the wave signal is dominated by convection rather than propagation, we can estimate $k_{\perp}=2 \pi f_{\mathrm{sc}} / V_{\mathrm{SW}}$. Figure 8 shows that the observed wave frequencies in the spacecraft frame vary from $0.2 \mathrm{~Hz}$ to almost $1 \mathrm{~Hz}$, but the spectrum in Figure 3 shows two peaks at $0.2 \mathrm{~Hz}$ and $0.8 \mathrm{~Hz}$. If we adopt these values as the natural range of the observations, we can compute $k_{\perp}=3 \times 10^{-3}$ and $1.2 \times 10^{-2} \mathrm{~km}^{-1}$, respectively, using $V_{\mathrm{SW}}=425 \mathrm{~km} \mathrm{~s}^{-1}$. From these values, the product of $k_{\perp}$ with the thermal proton gyroradius $\rho_{p}$ is computed and we obtain the two values $k v_{p} / \Omega_{\mathrm{cp}}=0.99$ and 3.96, which for all practical purposes are 1 and 4 , for $f_{\mathrm{sc}}=0.2$ and 0.8 , respectively. These would seem to satisfy the Bernstein mode condition $1<k v_{p} / \Omega_{\mathrm{cp}} \lesssim 3$. If these are Bernstein waves, then the wave frequency in the plasma frame is a multiple of the proton cyclotron frequency, $k v_{\mathrm{ph}}=\omega=n \Omega_{\mathrm{cp}}$. Since $k v_{p}=m \Omega_{\mathrm{cp}}$ where $m=1-4$, we can assert $v_{\mathrm{ph}}=\left(n \Omega_{\mathrm{cp}}\right) /\left(m \Omega_{\mathrm{cp}} / v_{p}\right)=(n / m) v_{p}$. Since $v_{p} \sim 10 \mathrm{~km} \mathrm{~s}^{-1}$, this places the phase speed of the wave in the right range for slowly propagating Bernstein waves.

Further evidence supporting our proton Bernstein mode interpretation for this wave event is the fact that these observations correspond to a relatively small proton beta $\left(0.05 \leqslant \beta_{p} \leqslant 0.4\right.$ as discussed in Section 2). Linear theory of the proton Bernstein instability driven by a suprathermal proton ring predicts that the maximum growth rate should arise near $\beta_{p} \simeq 0.10$ (e.g., Figure 3 of Gary et al. 2011), and that the maximum growth rate is significantly reduced as $\beta_{p}$ increases above 0.25 .

This brings us to the question "Why are the waves seen only late in the day"? And the answer to that question seems to lie in the balance between two competing dynamics. First, $\beta_{p}$ is smaller during the early hours of Figure 1 which favors the growth of Bernstein waves. Therefore, we would expect to see them at this time. However, and second, the IMF at this time is less perpendicular to the flow than it is by hour 20:00 UT, resulting in PUIs with a stronger streaming speed along the mean field. This geometry is contrary to the desired ring beam needed to drive Bernstein waves. Last, we note that waves at these frequencies may come and go on very short timescales and we do not claim to have found every instance during the two days of data we studied. In the end, we lack sufficient insight into the problem to fully account for the existence of these waves and will appeal to numerical solutions of the instability once they have been developed. For now we can only observe that the ability to excite Bernstein waves rests on more than one solar wind parameter while all are changing during the time studied.

Table 2

Whistler versus Bernstein Wave Parameters

\begin{tabular}{lccc}
\hline \hline & Whistler & Bernstein & Observation \\
\hline$\omega$ & $\Omega_{\mathrm{LH}}<\omega<\Omega_{\mathrm{ce}}$ & $\Omega_{\mathrm{cp}}<\omega<\Omega_{\mathrm{LH}}$ & $1-4 \Omega_{\mathrm{cp}}$ \\
$k$ & $k v_{e} / \Omega_{\mathrm{ce}}<1$ & $1<k v_{p} / \Omega_{\mathrm{cp}}<$ few & $(3-12) \times 10^{-3} \mathrm{~km}{ }^{-1}$ \\
$\Theta_{k B_{0}}$ & All angles & $k_{\|} \ll k_{\perp}$ & $90^{\circ}$ \\
$\delta \mathbf{B}\left(k_{\perp}=0\right)$ & $\left(\delta B_{\|}\right)^{2} /\left(\delta B_{\text {total }}\right)^{2}=0$ & $0<\left(\delta B_{\|}\right)^{2}<\left(\delta B_{\perp}\right)^{2}<1$ & $60^{\circ} \leqslant \Theta_{\mathbf{d B}, \mathbf{B}_{0} \leqslant 90^{\circ}}$ \\
$\delta \mathbf{B}\left(k_{\|} \ll k_{\perp}\right)$ & $\left(\delta B_{\|}\right)^{2} \simeq\left(\delta B_{\perp}\right)^{2}$ & & \\
\hline
\end{tabular}




\section{DISCUSSION}

It would be desirable to observe the more customary lowfrequency waves normally associated with PUIs during the event reported here. We do not observe them, but there are potential reasons for this. For example, their growth rates are generally slower and they favor quasi-radial IMF directions. It is not well understood why these low-frequency waves are not seen more often and we suspect that dynamics such as the turbulent cascade (Joyce et al. 2010) and related processes (Gamayunov et al. 2010) limit the observability of low-frequency waves. We do not consider the absence of low-frequency waves during this event to be a significant criticism of our interpretation.

It is the nature of single-spacecraft measurements that they are fundamentally frequency-based in the spacecraft frame. When a plasma frame wave period or inherent timescale for a fluctuation can be assumed to be longer than the convection timescale, we can relate spacecraft frame frequencies to reduced spatial scales along the line of convection (sunward). Anything more is speculation using known or suggested physics. We have interpreted the observations using the two modes most likely to arise in this situation: whistlers and Bernstein modes. We have concluded that the Bernstein mode better fits the observations and is more consistent with the hypothesized and expected source of PUIs. This interpretation is based largely on their observed frequency in the spacecraft frame as well as their linear polarization. Parallel propagating whistler modes of the type that would arise from an electron beam are ruled out on the basis of the waves not being circularly polarized. Whistler waves propagating at large angles to the IMF are ruled out, in large part, by the fact that half of the magnetic fluctuations show no projection onto the mean IMF. A likelihood and consistency argument is the best that we can do at this time. Numerical instability studies are underway and will be presented at a later date. These will shed some additional light on the interpretation.

We would be neglectful if we did not mention two other possible interpretations. The first is generation of ion cyclotron waves via the ion ring beam as mentioned at the top of Section 3. Podesta \& Gary (2011) believe they have found examples of these waves in the Ulysses data. Our initial scaling of this instability suggests a lower spacecraft frame frequency, but within the range of credibility and requiring additional consideration. The second would be spacecraft noise. The Voyager magnetometer data have not received a great deal of attention at these frequencies and the possibility of spacecraft noise has not been conclusively ruled out. The above hodograms do indicate a common line of polarization for the waves. Since the spacecraft is not spinning, the identification of additional events would hopefully resolve this ambiguity. Such identification has proven difficult and is problematic. It is expected that the PUI source will grow stronger as we examine data further from the Sun, but telemetry rates are reduced. This, the difficulty in processing the data, and competition for low- $\beta$ events without contamination by the Jovian tail greatly complicate the search for additional events.

\section{SUMMARY AND CONCLUSIONS}

We report a period of solar wind magnetic field observations revealing oscillations at spacecraft frame frequencies from 0.2 to $1 \mathrm{~Hz}$. We find the most plausible explanation to be that these are Bernstein waves excited by newly ionized interstellar PUIs gyrating in the perpendicular magnetic field. Whistler waves originating from PUIs, Jupiter, or a distant shock are ruled out by the wave properties and the mean field orientation. Bernstein waves are a rare observation in the solar wind and seldom discussed within that context, but we suggest they may be a fairly common observation in the outer heliosphere. An exhaustive search of high-resolution magnetic field data, although beyond the limits of this effort, may validate our claim. It is also possible that the instability and associated waves discussed here could provide a significant contribution to the heating of the solar wind in the outer heliosphere by PUIs (Zhou \& Matthaeus 1990; Zank et al. 1996; Matthaeus et al. 1999; Smith et al. 2001, 2006; Isenberg et al. 2003; Isenberg 2005; Breech et al. 2005, 2008; $\mathrm{Ng}$ et al. 2010) as well as the scattering of PUIs.

This work was supported in part by NASA Guest Investigator grant NNX07AH75G and NSF grant ATM0635863. C.W.S. is supported by Caltech subcontract 44A1085631 to the University of New Hampshire in support of the ACE/MAG instrument. Part of the ACE mandate is to better understand the role of pickup ions in the heliosphere. Portions of this research were carried out at the Jet Propulsion Laboratory, California Institute of Technology, under a contract with the National Aeronautics and Space Administration. C.J.J. was an undergraduate in the Physics program at UNH at the time this work was performed and is now a graduate student working within that same program. Perry Gray died unexpectedly just two weeks after this paper was first submitted to the journal. He was a bright and creative scientist who made lifelong friends everywhere he went. He will be greatly missed by friends and colleagues alike.

\section{REFERENCES}

Behannon, K. W., Acuña, M. H., Burlaga, L. F., et al. 1977, Space Sci. Rev., 21, 235

Breech, B., Matthaeus, W. H., Minnie, J., et al. 2005, Geophys. Res. Lett., 32, L06103

Breech, B., Matthaeus, W. H., Minnie, J., et al. 2008, J. Geophys. Res., 113, A08105

Bzowski, M., Möbius, E., Tarnopolski, S., Izmodenov, V., \& Gloeckler, G. 2009, Space Sci. Rev., 143, 177

Florinski, V., Zank, G. P., Heerikhuisen, J., Hu, Q., \& Khazanov, I. 2010, ApJ, 719, 1097

Gamayunov, K., Zhang, M., \& Rassoul, H. 2010, ApJ, 725, 2251

Gary, S. P., Hinata, S., Madland, C. D., \& Winske, D. 1986, Geophys. Res. Lett., 13,1364

Gary, S. P., \& Madland, C. D. 1988, J. Geophys. Res., 93, 235

Gary, S. P., Madland, C. D., Omidi, N., \& Winske, D. 1988, J. Geophys. Res., 93, 9584

Gary, S. P., Smith, C. W., Lee, M. A., Goldstein, M. L., \& Forslund, D. W. 1984, Phys. Fluids, 27, 1852

Gary, S. P., Liu, K., \& Winske, D. 2011, J. Geophys. Res., 116, A08215

Gary, S. P., Liu, K., Winske, D., \& Denton, R. E. 2010, J. Geophys. Res., 115, A12209

Gloeckler, G., Fisk, L. A., \& Geiss, J. 1997, Nature, 386, 374

Gray, P. C., Smith, C. W., Matthaeus, W. H., \& Otani, N. F. 1996, Geophys. Res. Lett., 23, 113

Isenberg, P. A. 2005, ApJ, 623, 502

Isenberg, P. A., Smith, C. W., \& Matthaeus, W. H. 2003, ApJ, 592, 564

Joyce, C. J., Smith, C. W., Isenberg, P. A., Murphy, N., \& Schwadron, N. A. 2010, ApJ, 724, 1256

Lee, M. A., \& Ip, W.-H. 1987, J. Geophys. Res., 92, 11,041

Liu, K., Gary, S. P., \& Winske, D. 2011, J. Geophys. Res., 116, A07212

Matthaeus, W. H., Zank, G. P., Smith, C. W., \& Oughton, S. 1999, Phys. Rev. Lett., 82, 3444

McClements, K. G., \& Dendy, R. O. 1993, J. Geophys. Res., 98, 11,689

McClements, K. G., Dendy, R. O., \& Lashmore-Davies, C. N. 1994, J. Geophys. Res., 99, 23,685

Murphy, N., Smith, E. J., Tsurutani, B. T., Balogh, A., \& Southwood, D. J. 1995, Space Sci. Rev., 72, 447

Ng, C. S., Bhattacharjee, A., Munsi, D., Isenberg, P. A., \& Smith, C. W. 2010, J. Geophys. Res., 115, A02101 
Perraut, S., Roux, A., Robert, P., et al. 1982, J. Geophys. Res., 87, 6219

Podesta, J. J., \& Gary, S. P. 2011, ApJ, 734, 15

Ruciński, D., Cummings, A. C., Gloeckler, G., et al. 1996, Space Sci. Rev, 78, 73

Smith, C. W., Goldstein, M. L., Matthaeus, W. H., \& Viñas, A. F. 1984, J. Geophys. Res., 89, 9159

Smith, C. W., Isenberg, P. A., Matthaeus, W. H., \& Richardson, J. D. 2006, ApJ, 638,508
Smith, C. W., Matthaeus, W. H., Zank, G. P., et al. 2001, J. Geophys. Res., 106, 8253

Wong, H. K., \& Goldstein, M. L. 1987, J. Geophys. Res., 92, 12,419

Wong, H. K., \& Goldstein, M. L. 1988, J. Geophys. Res., 93, 4110

Wong, H. K., \& Smith, C. W. 1994, J. Geophys. Res., 99, 13,373

Wu, C. S., \& Davidson, R. C. 1972, J. Geophys. Res., 77, 5399

Zank, G. P., Matthaeus, W. H., \& Smith, C. W. 1996, J. Geophys. Res., 101, 17,093

Zhou, Y., \& Matthaeus, W. H. 1990, J. Geophys. Res., 95, 10,291 